\title{
Four years of experience with carbapenem-resistant Gram-negative bacteria in two tertiary care hospitals in Crete, Greece
}

\author{
C Tsioutis ${ }^{1,2 *}$, S Karageorgos ${ }^{2}$, S Stratakou ${ }^{2}$, E Bolikas ${ }^{3}$, E Astrinaki ${ }^{1}$, A Messaritaki ${ }^{1}$, M Adami ${ }^{2}$, A Georgiladakis ${ }^{4}$, \\ A Kassimati ${ }^{5}$, A Gikas ${ }^{1,2}$
}

From 3rd International Conference on Prevention and Infection Control (ICPIC 2015)

Geneva, Switzerland. 16-19 June 2015

\section{Introduction}

Carbapenem-resistant gram-negative bacteria (CRGNB, Acinetobacter baumannii, Klebsiella pneumoniae, Pseudomonas aeruginosa)) are important nosocomial pathogens in Greece.

\section{Objectives}

To describe the epidemiology of CRGNB in two tertiary care hospitals in Crete, Greece.

\section{Methods}

Analysis of infection control records from June 2011 to December 2014 of 450-bed Venizelion Hospital and 750-bed University Hospital, both being referral centres for Southern Greece. Consecutive patients with CRGNB isolation (only first CRGNB per patient) were recorded. Data presented as no.(\%) or mean \pm SD.

\section{Results}

A total of 1537 cases with CRGNB were detected: 582 A. baumannii, $510 \mathrm{~K}$. pneumoniae and 445 P. aeruginosa. Mean patient age was $63.6 \pm 18.7$ years. The greatest burden was in ICU (41.4\%) and medical wards (36.7\%). Respiratory specimens constituted the most frequent source of A. baumannii (56.3\%) and P. aeruginosa (43.6\%); urine samples were the most frequent source of $K$. pneumoniae (28.8\%). In-hospital mortality (37.7\%) was similar among the 3 pathogen groups; however, ICU patients with CRGNB had higher mortality rates compared to other departments $(53.5 \%$ vs $26.6 \%, \mathrm{p}<0.001)$. Similarly, ICU patients had longer hospital stay after CRGNB isolation compared to other departments (median 21 vs 8 days, $\mathrm{p}<0.001)$.

\section{Conclusion}

This study shows that the burden of CRGNB is much greater in ICUs, accounting for significant morbidity and mortality. Therefore, in Greek hospitals where CRGNB infections are a major problem, infection control measures should mainly focus in critical care departments.

\section{Disclosure of interest}

None declared.

\section{Authors' details}

${ }^{1}$ Infection Control Unit, University Hospital of Heraklion, Heraklion, Crete, Greece. ${ }^{2}$ Internal Medicine / Infectious Diseases, University Hospital of Heraklion, Heraklion, Crete, Greece. ${ }^{3}$ Infection Control Unit, Venizelion General Hospital, Heraklion, Crete, Greece. ${ }^{4}$ Department of Microbiology, University Hospital of Heraklion, Heraklion, Crete, Greece. ${ }^{5}$ Department of Microbiology, Venizelion General Hospital, Heraklion, Crete, Greece.

Published: 16 June 2015

doi:10.1186/2047-2994-4-S1-P130

Cite this article as: Tsioutis et al:: Four years of experience with carbapenem-resistant Gram-negative bacteria in two tertiary care hospitals in Crete, Greece. Antimicrobial Resistance and Infection Control 2015 4(Suppl 1):P130. 